\title{
Addition of Camrelizumab to Transarterial Chemoembolization in Hepatocellular Carcinoma With Untreatable Progression
}

\author{
Yanqiao Ren \\ Wuhan Union Hospital \\ Ziyi Liu \\ Wuhan Union Hospital \\ Joyman Makamure \\ Wuhan Union Hospital \\ Xuefeng Kan \\ Wuhan Union Hospital \\ Songlin Song \\ Wuhan Union Hospital \\ Yiming Liu \\ Wuhan Union Hospital \\ Kun Qian \\ Wuhan Union Hospital \\ Chuansheng Zheng \\ Wuhan Union Hospital \\ Bin Liang ( $\sim$ bliang@hust.edu.cn ) \\ Wuhan Union Hospital
}

\section{Research Article}

Keywords: camrelizumab, transarterial chemoembolization, untreatable progression, progression-free survival, adverse events

Posted Date: August 9th, 2021

DOl: https://doi.org/10.21203/rs.3.rs-771504/v1

License: (c) (1) This work is licensed under a Creative Commons Attribution 4.0 International License.

Read Full License 


\section{Abstract}

Purpose: To evaluate the efficacy and safety of camrelizumab addition to transarterial chemoembolization (TACE) in treatment of hepatocellular carcinoma (HCC) with TACE-related untreatable progression (UP).

Methods: Patients with HCC who received addition of camrelizumab due to UP after initial TACE treatment were enrolled at our institution between May 2019 and January 2021. Patients were assessed for tumor response, progression-free survival (PFS), and adverse events. Risk factors for PFS were evaluated with logistic regression analysis.

Results: A total of 41 patients were included. The objective response rate and disease control rate were $24.4 \%$ and $61.0 \%$ at $2-3$ months, and $12.2 \%$ and $58.5 \%$ at 6 months, respectively. The median PFS of the patients were 6 months ( $95 \%$ Cl: 3.8 months, 8.2 months). Of the 41 patients, 23 patients received camrelizumab combined with TACE (hereafter, camrelizumab-TACE), in whom 52 combined TACE procedures were performed, with a median of 2 procedures (range, 1-6) per patient. The remaining 18 patients received camrelizumab alone due to TACE contraindications. Multivariable analysis indicated that camrelizumab-TACE was an independent prognostic factor for PFS. Subgroup analysis showed a median PFS of 8 months in the camrelizumab-TACE group and 3 months in the camrelizumab monotherapy group $(P<0.001)$. No treatment-related mortalities occurred. Seventeen patients $(41.5 \%)$ developed at least one type of adverse events after treatment with camrelizumab, with reactive cutaneous capillary endothelial proliferation ( $n=14,34.1 \%)$ being the most common adverse events.

Conclusions: Addition of camrelizumab to TACE offered an effective and safe treatment for HCC with UP.

\section{Introduction}

Globally, and especially in China, the prognosis of patients with hepatocellular carcinoma (HCC) remains a depressing issue. It is currently one of the most frequent tumors in the world and the second common cause of cancer-related death [1]. Due to insufficient liver reserve and donor shortage, some curative methods, such as hepatectomy and liver transplantation, are only suitable for a small number of patients [2]. As a result, only less than $30 \%$ of HCC patients can benefit from curative therapies [3].Currently, transarterial chemoembolization (TACE) as a palliative therapy has been recognized as the most commonly used treatment for unresectable HCC [4]. However, some tumor cells may still survive after a session of TACE, and low rates of complete response (CR) after TACE have been reported, ranging from $23-27 \%[5,6]$. Hence, repeated TACE is the most commonly used method for local or intrahepatic residual HCC [7].

However, the efficacy of TACE decreased significantly as the number of TACE procedures increased, with rates of progressive disease (PD) after the first, second, third, and fourth TACE procedures reported to be $18,21,25$, and $27 \%$, respectively [8]. Based on this phenomenon, the Japan Society of Hepatology (JSH) first proposed the concept of TACE failure/refractoriness in 2010 [9], and updated it in 2014 [10]. It should 
be noted, however, that the JSH standard appears to be problematic because new intrahepatic tumors are far from the target territory, and while new intrahepatic tumors represent PD, additional TACE therapy is not contraindined. Thus, a new concept, untreatable progression (UP), was proposed to determine TACE discontinuation $[1,11,12]$, which includes both major progression (such as extensive hepatic involvement, extrahepatic metastasis, or vascular invasion) and minor intrahepatic progression associated with impaired liver function and performance status. Currently, for those patients who have UP after TACE, treatment is tricky and there is still no consensus, which may create an incentive to try other therapies and approaches.

Immune checkpoint inhibitors (ICls) that reverse immune exhaustion have been shown to be effective in $\mathrm{HCC}$, which were used alone and in combination with TACE in treatment of intermediate and advanced HCC [13-15]. Recently, camrelizumab, an anti-PD-1 monoclonal antibody, was approved in China as a second-line treatment for unresectable HCC. Camrelizumab has been shown to be comparable to the efficacy of nivolumab and pembrolizumab in the treatment of advanced HCC [16]. Accordingly, we hypothesized that camrelizumab might be effective in treating UP HCC after TACE, but no studies have been reported so far. Meanwhile, tumors with a low mutation burden and fewer neoantigens are generally less immunogenic, so they have little response to immunotherapy. TACE has been reported to promote tumor-specific $\mathrm{CD}^{+} \mathrm{T}$ cell response by killing $\mathrm{HCC}$ cells and causing the release of tumor-associated antigens [17]. Thus, the application of camrelizumab after TACE treatment may be able to achieve promising outcomes in UP HCC. However, to our knowledge, no studies have been reported on the treatment of UP HCC with camrelizumab. Thus, the present study reports the safety and efficacy of camrelizumab in the treatment of UP HCC patients.

\section{Material And Methods}

\section{Patients}

A retrospective analysis was conducted of all HCC patients who received TACE plus camrelizumab at Union Hospital, Tongji Medical College, Huazhong University of Science and Technology between May 2019 and January 2021. The criteria for patient selection included: (1) patients aged > 18 years with HCC confirmed by pathological or clinical diagnosis according to EASL criteria [1]; (2) patients who received additional camrelizumab due to UP after initial TACE treatment $[1,11,12]$; (3) Child-Pugh class A or B without ascites; and (4) an Eastern Cooperative Oncology Group (ECOG) performance status of 0 or 1. Exclusion criteria were: (1) concurrent ablation, resection, radiotherapy, or systemic therapies for HCC; (2) discontinuation of camrelizumab due to serious adverse events (AEs); and (3) loss to follow-up.

The present retrospective, single-center study was conducted in accordance with the principles of the Declaration of Helsinki and all procedures performed in this study were approved by the Ethics Committee of Union hospital, Tongji Medical College, Huazhong University of Science and Technology. Written informed consent was obtained from all patients prior to treatment. 


\section{TACE procedure}

TACE was performed according to our institutional standard protocol and has been previously reported [18]. Briefly, after superselective catheterization of tumor-feeding arterials with coaxial microcatheter (Progreat, Terumo, Tokyo, Japan), a solution of multiple chemotherapeutic agents (epirubicin 40-60 mg, cisplatin, oxaliplatin or lobaplatin $50-100 \mathrm{mg}$, and 5-Fu or floxuridine $1.0 \mathrm{~g}$ ) or single chemotherapeutic agent (epirubicin 40-60 mg) with ethiodized oil was subsequently injected. This was followed by injection of gelatin sponge particles (350-560 $\mu \mathrm{m}$ or 560-710 $\mu \mathrm{m}$, Alicon, Hangzhou, China). Embolization was performed under fluoroscopic guidance until the stasis of arteries flow was occurred. Reexamination angiography of the hepatic artery was performed to validate the devascularization.

UP after TACE was defined as progression associated with a clinical profile that prevents retreatment, which includes at least one of the following situations: situation I, targeted tumor failed to achieve objective response after at least two initial TACE treatments; situation II, local tumor progression or new intrahepatic tumor did not achieve objective response after another TACE session; situation III, presence of significant progression, including substantial liver involvement, vascular invasion or extrahepatic metastasis; and situation IV, presence of hepatic dysfunction (Child-Pugh C) or ECOG performance status $>2$ that contraindicates TACE therapy [11].

\section{Camrelizumab therapy}

The administration of camrelizumab was initiated after UP in HCC patients. Camrelizumab was administered intravenously at a dose of $200 \mathrm{mg}$ every 3 weeks. If patients developed serious AEs, the drug was interrupted or discontinued and symptomatic treatment such as glucocorticoids or immunesuppressant agents were administered, depending on the severity and the affected organs. For patients who required multiple TACE procedures, the camrelizumab was administered within 2 weeks after TACE.

\section{Follow-up and evaluation}

All patients were followed up until June 2021. Laboratory examinations, abdominal contrast-enhanced CT or MR were performed every 6 to 8 weeks after initial camrelizumab treatment. Laboratory tests mainly included blood routine and tests of heart, liver and kidney function. Follow-up CT or MR at 2-3 and 6 months after initial camrelizumab treatment were compared with pretreatment imaging to determine objective response rate (ORR) and disease control rate (DCR) according to Modified Response Evaluation Criteria in Solid Tumors (mRECIST) [19]. ORR was defined as a CR or partial response (PR). DCR represented CR, PR or stable disease (SD).

Progression-free survival (PFS), defined as the time interval from initial camrelizumab treatment to the date of progression for patients who displayed radiologic evidence of disease progression or the date of death or last follow-up, was the primary endpoint of this study. AEs were recorded and assessed by the Common Terminology Criteria for Adverse Events (CTCAE) Version 5.0. In addition, postembolization 
syndrome, such as fever, pain, nausea and vomiting, is not considered an AE in itself, but rather an expected outcome of embolization therapy [20].

\section{Statistical Analyses}

All analyses were performed by using SPSS software, Version 24.0 (IBM, Armonk, New York). Discrete variables were presented as numbers with percentages, and quantitative data were presented as mean \pm standard deviation. PFS were calculated by using Kaplan-Meier method. The $95 \%$ confidence interval (Cl) was calculated for median PFS and hazard ratio (HR). Risk factors for PFS were evaluated with logistic regression analysis. All statistical tests were two tailed and $P<0.05$ indicated a statistically significance.

\section{Results}

\section{Patient characteristics}

A total of $89 \mathrm{HCC}$ patients received additional camrelizumab due to UP after initial TACE treatment in our hospital between May 2019 and January 2021. Of those, 28 were excluded due to concurrent ablation, resection, radiotherapy, or systemic therapies, 11 were excluded due to camrelizumab discontinuation, and 9 patients were excluded because they were lost to follow-up. Finally, 41 patients were included in this study (Figure 1). The situation I, II, and III of UP occurred in 11, 13, and 17 patients, respectively. A total of 150 TACE sessions were performed before the addition of camrelizumab, with a median TACE procedure of 3 (range, 1-19) per patient. The overall patient characteristics are shown in Table 1. In addition, 15 patients (36.6\%) died during the observation period of the study.

\section{Addition of camrelizumab to TACE}

The 41 patients received 280 camrelizumab therapy sessions, with a median of 5 sessions (range, 1-17) per patient. Twenty-three patients received camrelizumab combined with TACE (hereafter, camrelizumabTACE), in whom 52 combined TACE procedures were performed, with a median of 2 procedures (range, 16) per patient. The remaining 18 patients received camrelizumab alone due to TACE contraindications.

\section{Tumor response}

Imaging results 2-3 months after intervention indicated that 1 patient (2.4\%) achieved CR, 9 patients (22.0\%) achieved PR, and 15 patients (36.6\%) achieved SD. Thus, the ORR is $24.4 \%$ and the DCR is $61.0 \%$. Imaging results 6 months after intervention showed CR in 1 patient (2.4\%), PR in 4 patients (9.8\%), and SD in 19 patients (46.3\%). Therefore, ORR was $12.2 \%$ and DCR was $58.5 \%$.

\section{Progression-free survival}

The median follow-up period from camrelizumab initiation to the study's end point was 7 months (range, 4-14 months). Of the 41 patients, 33 (80.5\%) develop disease progression after addition of camrelizumab. The median PFS in this study was 6 months ( $95 \%$ Cl: 3.8 months, 8.2 months) (Figure 2). 
Univariate analysis (Table 1) indicated that combination therapy and platelet-to-lymphocyte ratio (PLR) were significantly associated with PFS $(P<0.05)$. At multivariable analysis (Table 2$)$, combination therapy (camrelizumab-TACE) was significantly in connection with better PFS $(P=0.003)$.

\section{Subgroup analysis by treatment modalities}

In this study, 23 patients received camrelizumab-TACE, and 18 patients received camrelizumab alone. The median PFS was 8 months (95\% Cl: 5.8 months, 10.2 months) and 3 months ( $95 \%$ Cl: 2.7 months, 3.3 months) in the camrelizumab-TACE and camrelizumab subgroups (Figure 3), respectively, with a statistically significant difference between them $(P<0.001)$.

\section{Adverse events}

Twenty-one (51.2\%) patients developed post-embolization syndrome, including fever $(n=18)$, abdominal pain $(n=15)$, nausea and vomiting $(n=10)$ within one week after TACE. After symptomatic treatment during hospitalization, the symptoms of all patients were relieved or significantly improved. In addition, there were no TACE related severe AEs such as liver abscess and biloma, and no allergic events occurred during camrelizumab injection.

During the follow-up period, 17 (41.5\%) patients developed at least one type of AEs after treatment with camrelizumab (Table 3), and no patients developed severe AEs (more than grade III). Grade I/II AEs included reactive cutaneous capillary endothelial proliferation (RCCEP) $(n=14)$, hypothyroidism $(n=6)$, asthenia $(n=2)$, rash $(n=1)$, myositis $(n=1)$, and pneumonitis $(n=1)$. Of noted, the 2 patients with pneumonia and myositis who received glucocorticoids and were temporarily interrupted from camrelizumab demonstrated significant improvement in symptoms, and then resumed camrelizumab therapy. No treatment-related mortalities occurred.

\section{Discussion}

Currently, the treatment of HCC patients with UP after TACE remains a thorny issue. Changes in T-cell populations after TACE have been demonstrated, which provides impetus for the exploration of immunotherapy after TACE [21]. The present study indicated that additional camrelizumab therapy is an effective and safe in the treatment of HCC patients with UP after initial TACE treatment.

Patients with HCC who develop UP after TACE treatment have a poor prognosis, and these patients are reported to be good candidates for tyrosine kinase inhibitors [22-24]. Lee et al. investigated 54 patients receiving sorafenib who met the criteria of TACE failure as defined by the European and Japanese international guidelines and showed a median PFS of 3.2 months [22]. Similarly, another study [23] comparing sorafenib with hepatic arterial infusion of cisplatin in HCC patients who refractory to TACE showed a median time to progression of 3.9 months for sorafenib and 2 months for cisplatin, respectively. In addition, the efficacy of lenvatinib in patients with intermediatestage HCC refractory to TACE was reported by Shimose et al [24]. The median PFS in the lenvatinib group was 5.8 months, higher 
than that in the sorafenib group (3.2 months) and TACE group (2.4 months). However, the study only included patients with intermediatestage HCC and lacked results for patients with advanced HCC. In this study, additional camrelizumab therapy achieved a median PFS of 6 months in patients with UP after TACE, which seems to imply that ICls represent effective systemic therapy for patients with TACE failure.

In terms of tumor response, camrelizumab also had comparable outcomes for HCC with UP after TACE treatment. Previous studies have shown that nivolumab as a first-line and second-line treatment for advanced HCC patients has an ORR of $15 \%$ [25] and 20\% [13], respectively. Similarly, pembrolizumab as a first-line and second-line treatment for advanced HCC patients has an ORR of 17\% [26] and $18.4 \%$ [27], respectively. Meanwhile, the ORR of camrelizumab in advanced HCC patients who had failed chemotherapy or sorafenib treatment was $14.7 \%$ [16]. The results of this study showed that ORR and DCR were $24.4 \%$ and $61.0 \%$, respectively, suggesting that camrelizumab therapy may achieve comparable tumor control in HCC patients with TACE failure.

In this study, multivariable analysis indicated that camrelizumab-TACE was an independent prognostic factor for PFS. Additional subgroup analysis demonstrated that the camrelizumab-TACE therapy significantly improved median PFS compared with camrelizumab monotherapy. It is reported that TACE can promote tumor-specific CD $8^{+} \mathrm{T}$ cell response by killing HCC cells and causing the release of tumorassociated antigens [17]. As suggested by the theoretical advantages, both Zhan et al [15] and Marinelli et al [14] demonstrated the efficacy of transarterial embolization combined with ICls in the treatment of HCC. Similarly, this study also confirmed that TACE combined with ICI was superior to ICl monotherapy. These outcomes should promote further prospective studies evaluating combination TACE and systemic immunotherapy for the treatment of HCC.

Recent studies have shown the inflammation ratio of PLR may potentially serve as a quantitative biomarker for individual tumor characteristics [28, 29]. Isabel et al [30] investigated inflammatory biomarkers in patients with HCC treated with TACE and found that high baseline PLR predicted poorer tumor response and shorter PFS. Schobert et al [31] and Fan et al [32] also reported that high PLR is associated with poorer overall survival and metastasis in patients with HCC treated with TACE. In this study, although the univariate analysis showed that PLR was associated with PFS, multivariable analysis did not indicate it was an independent prognostic factor affecting PFS. This may be related to the small sample size of this study. Additional study with larger sample size is warranted to validate risk factors to UP after TACE.

This study found that $39.6 \%$ of patients had AEs, but no patients had AEs of grade III or higher. Meanwhile, camrelizumab had a lower incidence of AEs in HCC patients with UP after TACE compared with sorafenib $[23,33]$. Unlike nivolumab and pembrolizumab, RCCEP is more common after camrelizumab treatment [34]. RCCEP incidence after camrelizumab monotherapy has been reported in 76.7-97.3\% in other tumors, but no grade III RCCEP was reported [27, 35]. Similar to the study of Qin et al., the most common AEs in this study was also RCCEP [16]. However, the AEs incidence of grade III or 
more with camrelizumab seemed to be lower than that with other ICls [14, 15], suggesting that camrelizumab is safe for the treatment of HCC with UP after TACE.

The retrospective study had several limitations. First, the present study was conducted in a single institution with a small sample size, and therefore, a multicenter prospective randomized trial is needed. Second, most patients were still alive at the time of data collection and were followed up for a relatively short period of time and did not obtain the median OS for this study. Thus, it is necessary to explore the long-term efficacy of patients. Lastly, we did not include a control group of HCC patients with UP after TACE who received sorafenib or other forms of systemic therapies. Therefore, further comparative studies are needed to elucidate the clinical efficacy of camrelizumab in the treatment of UP HCC.

\section{Conclusions}

The present study first reported camrelizumab in the treatment of HCC patients with UP after TACE, and the results indicated that camrelizumab has an acceptable safety profile and promising tumor control. Meawhile, this study also demonstrated the superiority of camrelizumab-TACE verse camrelizumab monotherapy. The results of this study should prompt further prospective studies to evaluate the combination of TACE and ICls in the treatment of HCC.

\section{Abbreviations}

HCC, hepatocellular carcinoma; TACE, transarterial chemoembolization; CR, complete response; PD, progressive disease; JSH, Japan Society of Hepatology; UP, untreatable progression; ICls, immune checkpoint inhibitors; ECOG, Eastern Cooperative Oncology Group; AEs, adverse events; ORR, objective response rate; DCR, disease control rate; mRECIST, Modified Response Evaluation Criteria in Solid Tumors; PR, partial response; SD, stable disease; PFS, progression-free survival; CTCAE, Common Terminology Criteria for Adverse Events; $\mathrm{Cl}$, confidence interval; HR, hazard ratio.

\section{Declarations}

\section{Acknowledgements}

This work was supported by grant from National Nature Science Foundation of China (No.81771950, No.81471765, and No.81873919), Fundamental Research Funds for the Central Universities (2021yjsCXCY101).

\section{Compliance with ethical standards}

Conflicts of Interest All authors (Yanqiao Ren, Ziyi Liu, Joyman Makamure, Xuefeng Kan, Songlin Song, Yiming Liu, Kun Qian, Chuansheng Zheng, Bin Liang) declare that they have no conflict of interest. 
Ethics approval statement This retrospective study was approved by the institutional review board of the Union Hospital, Tongji Medical college, Huazhong University of Science and Technology.

Informed consent Written informed consent was obtained from all patients prior to treatment.

\section{Funding}

This work was supported by grant from National Nature Science Foundation of China (No.81771950, No.81471765, and No.81873919), Fundamental Research Funds for the Central Universities (2021yjsCXCY101).

\section{Authors' contributions}

YQR, ZYL and MJ collected the patients' data. YQR and ZYL drafted the manuscript. SLS, YML, KQ, CSZ and $b$ revised the manuscript. YQR, ZYL and XFK analyzed and interpreted the data. XFK and BL made substantial contributions to the conception of the work. CSZ and BL made substantial contributions to the design of the work and have revised the manuscript substantively. All authors read and approved the final manuscript.

\section{Availability of data and material}

The datasets used in this study are available from the corresponding author upon reasonable request.

\section{References}

1. European Association for the Study of the Liver. EASL clinical practice guidelines: management of hepatocellular carcinoma. J Hepatol. 2018;69:182-236.

2. Kim JW, Kim JH, Sung KB, et al. Transarterial chemoembolization vs. radiofrequency ablation for the treatment of single hepatocellular carcinoma $2 \mathrm{~cm}$ or smaller. Am J Gastroenterol. 2014;109:123440.

3. Llovet JM, Bruix J. Systematic review of randomized trials for unresectable hepatocellular carcinoma: chemoembolization improves survival. Hepatology. 2003;37:429-42.

4. Sheta E, El-Kalla F, El-Gharib M, et al. Comparison of single-session transarterial chemoembolization combined with microwave ablation or radiofrequency ablation in the treatment of hepatocellular carcinoma: a randomized-controlled study. Eur J Gastroenterol Hepatol. 2016;28:1198-203.

5. Song MJ, Chun HJ, Song DS, et al. Comparative study between doxorubicineluting beads and conventional transarterial chemoembolization for treatment of hepatocellular carcinoma. J Hepatol. 2012;57:1244-50.

6. Nicolini A, Martinetti L, Crespi S, et al. Transarterial chemoembolization with epirubicin-eluting beads versus transarterial embolization before liver transplantation for hepatocellular carcinoma. J Vasc Interv Radiol. 2010;21:327-32. 
7. Takayasu K, Arii S, Ikai I, et al. Prospective cohort study of transarterial chemoembolization for unresectable hepatocellular carcinoma in 8510 patients. Gastroenterology. 2006;131:461-9.

8. Peck-Radosavljevic M, Kudo M, Raoul JL, et al. Outcomes of patients (pts) with hepatocellular carcinoma (HCC) treated with transarterial chemoembolization (TACE): Global OPTIMIS final analysis. J Clin Oncol. 2018;36:4018.

9. Kudo M, Izumi N, Kokudo N, et al. HCC Expert Panel of Japan Society of Hepatology. Management of hepatocellular carcinoma in Japan: Consensus-Based Clinical Practice Guidelines proposed by the Japan Society of Hepatology (JSH) 2010 updated version. Dig Dis. 2011;29:339-64.

10. Kudo M, Matsui O, Izumi N, et al. Liver Cancer Study Group of Japan. Transarterial chemoembolization failure/refractoriness: JSH-LCSGJ criteria 2014 update. Oncology. 2014;87(Suppl 1):22-31.

11. Forner A, Gilabert M, Bruix J, Raoul JL. Treatment of intermediate-stage hepatocellular carcinoma. Nat Rev Clin Oncol. 2014;11:525-35.

12. Raoul JL, Gilabert M, Piana G. How to define transarterial chemoembolization failure or refractoriness: a European perspective. Liver Cancer. 2014;3:119-24.

13. El-Khoueiry AB, Sangro B, Yau T, et al. Nivolumab in patients with advanced hepatocellular carcinoma (CheckMate 040): an open- label, non- comparative, phase 1/2 dose escalation and expansion trial. Lancet. 2017;389:2492-502.

14. Marinelli B, Cedillo M, Pasik SD, et al. Safety and Efficacy of Locoregional Treatment during Immunotherapy with Nivolumab for Hepatocellular Carcinoma: A Retrospective Study of 41 Interventions in 29 Patients. J Vasc Interv Radiol. 2020;31:1729-38.e1.

15. Zhan C, Ruohoniemi D, Shanbhogue KP, et al. Safety of Combined Yttrium-90 Radioembolization and Immune Checkpoint Inhibitor Immunotherapy for Hepatocellular Carcinoma. J Vasc Interv Radiol. 2020;31:25-34.

16. Qin S, Ren Z, Meng Z, et al. Camrelizumab in patients with previously treated advanced hepatocellular carcinoma: a multicentre, open-label, parallel-group, randomised, phase 2 trial. Lancet Oncol. 2020;21:571-80.

17. Chao J, Zhu Q, Chen D, et al. Case Report: Transarterial Chemoembolization in Combination With Tislelizumab Downstages Unresectable Hepatocellular Carcinoma Followed by Radical Salvage Resection. Front Oncol. 2021;11:667555.

18. Ren Y, Cao Y, Ma H, et al. Improved clinical outcome using transarterial chemoembolization combined with radiofrequency ablation for patients in Barcelona clinic liver cancer stage A or B hepatocellular carcinoma regardless of tumor size: results of a single-center retrospective case control study. BMC Cancer. 2019;19:983.

19. Lencioni R, Llovet JM. Modified RECIST (mRECIST) Assessment for Hepatocellular Carcinoma. Semin Liver Dis. 2010;30:52-60.

20. Gaba RC, Lokken RP, Hickey RM, et al. Quality Improvement Guidelines for Transarterial Chemoembolization and Embolization of Hepatic Malignancy. J Vasc Interv Radiol. 2017;28:1210- 
23.

21. Takaki H, Imai N, Contessa TT, et al. Peripheral blood regulatory T-cell and type 1 helper T-cell population decrease after hepatic artery embolization. J Vasc Interv Radiol. 2016;27:1561-8.

22. Lee S, Kang JH, Kim DY, et al. Prognostic factors of sorafenib therapy in hepatocellular carcinoma patients with failure of transarterial chemoembolization. Hepatol Int. 2017;11:292-9.

23. Ikeda M, Mitsunaga S, Shimizu S, et al. Efficacy of sorafenib in patients with hepatocellular carcinoma refractory to transcatheter arterial chemoembolization. J Gastroenterol. 2014;49:932-40.

24. Shimose S, Kawaguchi T, Tanaka M, et al. Lenvatinib prolongs the progression-free survival time of patients with intermediate-stage hepatocellular carcinoma refractory to transarterial chemoembolization: A multicenter cohort study using data mining analysis. Oncol Lett. 2020;20:2257-65.

25. Yau T, Park JW, Finn RS. et al. CheckMate 459: A randomized, multi-center phase III study of nivolumab (NIVO) vs sorafenib (SOR) as first-line (1L) treatment in patients (pts) with advanced hepatocellular carcinoma (aHCC). Ann Oncol. 2019;30:v874-5.

26. Zhu AX, Finn RS, Edeline J, et al. Pembrolizumab in patients with advanced hepatocellular carcinoma previously treated with sorafenib (KEYNOTE-224): a non-randomised, open-label phase 2 trial. Lancet Oncol. 2018;19:940-52.

27. Finn RS, Ryoo BY, Merle P, et al. Pembrolizumab As Second-Line Therapy in Patients With Advanced Hepatocellular Carcinoma in KEYNOTE-240: A Randomized, Double-Blind, Phase III Trial. J Clin Oncol. 2020;38:193-202.

28. Kuang DM, Zhao Q, Wu Y, et al. Peritumoral neutrophils link inflammatory response to disease progression by fostering angiogenesis in hepatocellular carcinoma. J Hepatol. 2011;54:948-55.

29. Bambace NM, Holmes CE. The platelet contribution to cancer progression. J Thromb Haemost. 2011;9:237-49.

30. Schobert IT, Savic LJ, Chapiro J, et al. Neutrophil-to-lymphocyte and platelet-to-lymphocyte ratios as predictors of tumor response in hepatocellular carcinoma after DEB-TACE. Eur Radiol. 2020;30:5663-73.

31. Shen Y, Wang H, Chen X, et al. Prognostic significance of lymphocyte-to-monocyte ratio and plateletto-lymphocyte ratio in patients with hepatocellular carcinoma undergoing transcatheter arterial chemoembolization and radiofrequency ablation. Onco Targets Ther. 2019;12:7129-37.

32. Fan W, Zhang Y, Wang Y, et al. Neutrophil-to-lymphocyte and platelet-to-lymphocyte ratios as predictors of survival and metastasis for recurrent hepatocellular carcinoma after transarterial chemoembolization. PLoS One. 2015;10:e0119312.

33. Lin PT, Teng W, Jeng WJ, et al. Add-on sorafenib is beneficial for hepatocellular carcinoma patients with transarterial chemoembolization refractoriness: a real-world experience. Eur J Gastroenterol Hepatol. 2020;32:1192-9.

34. Hwang SJ, Carlos G, Wakade D, et al. Cutaneous adverse events (AEs) of anti-programmed cell death (PD)-1 therapy in patients with metastatic melanoma: A single-institution cohort. J Am Acad 
Dermatol. 2016;74:455-61.

35. Huang J, Xu B, Mo H, et al. Safety, activity, and biomarkers of SHR-1210, an anti-pd-1 antibody, for patients with advanced esophageal carcinoma. Clin Cancer Res. 2018;24:1296-304.

\section{Tables}

Table 1 Patient demographics and clinical status at camrelizumab initiation $(n=41)$ and univariate analysis of prognostic factors for progression-free survival 
Characteristic

Patients with UP after TACE (No, \%; Mean HR (95\% Cl) $\pm \mathrm{SD})$

\section{Gender}

Male

$32(78.0 \%)$

1

Female

$9(22.0 \%)$

$0.945(0.404$,

0.897

2.213)

Age ( $y)$

$54.2 \pm 11.4$

$1.011(0.979$,

1.044)

0.503

ECOG

1

0

\section{BCLC stage}

C

B

Largest diameter of tumor

(cm)

$23(56.1 \%)$

1

$18(43.9 \%)$

$0.886(0.442$,
$1.775)$

0.732
1

$0.682(0.310, \quad 0.342$

1.501)

$8.0 \pm 4.9$

1.009 (0.941,

1.082)

0.799

Hepatitis

Hepatitis B

$35(85.4 \%)$

1

Other

$6(14.6 \%)$

1.240 (0.474,

3.243)

0.661

a-Fetoprotein level

$\varangle 400 \mathrm{ng} / \mathrm{mL}$

$20(48.8 \%)$

1

$\leq 400 \mathrm{ng} / \mathrm{ml}$

$21(51.2 \%)$

0.780 (0.390,

0.483

1.560)

\section{Child-Pugh score}

B

$5(12.2 \%)$

1

A

$36(87.8 \%)$

$1.213(0.367$,
$4.004)$

0.751

\section{Combination therapy}

Yes

No
$23(56.1 \%)$

$18(43.9 \%)$
1

$3.899(1.818, \quad 0.000$ 
TACE sessions before UP $\quad 3.7 \pm 3.4$

$1.036(0.934, \quad 0.500$

1.150)

\section{Tumor number}

$\begin{array}{llll}\geq 3 & 36(87.8 \%) & 1 & \\ <3 & 5(12.2 \%) & 1.361(0.469, & 0.570\end{array}$

\section{Ascites}

Present

$4(9.8 \%)$

1

Absent

$37(90.2 \%)$

$1.620(0.384$, 6.827)

0.511

\section{Extrahepatic spread}

Present

$13(31.7 \%)$

1

Absent

28 (68.3\%)

1.247 (0.579, 2.689)

0.573

Vascular invasion

Present

$18(43.9 \%)$

1

Absent

$23(56.1 \%)$

0.933 (0.469, 1.856)

0.844

*Interval time

$>2$ weeks

$16(39.0 \%)$

1

$<2$ weeks

$25(61.0 \%)$

$0.570(0.284$,

1.146)

0.115

\section{Baseline laboratory test result}

TB $(\mu \mathrm{mol} / \mathrm{L})$

$15.9 \pm 8.4$

1.031 (0.990,

1.072)

Albumin (g/L)

$35.1 \pm 4.0$

0.957 (0.883,

1.038)

PT(s)

$14.0 \pm 1.0$

1.025 (0.739,

1.421)

AST $(\mu \mathrm{mol} / \mathrm{L})$

$53.1 \pm 42.1$

1.002 (0.997,

1.008)

ALT $(\mu \mathrm{mol} / \mathrm{L})$

$38 \pm 19.6$

1.000 (0.983,

1.018)

0.138

0.999 (0.979,

0.292

Creatinine $(\mu \mathrm{mol} / \mathrm{L})$

$63.8 \pm 18.4$

0.883

0.394

0.992 
1.020)

\begin{tabular}{|c|c|c|c|}
\hline PLR & $181.9 \pm 117.6$ & $\begin{array}{l}1.003(1.000 \\
1.005)\end{array}$ & 0.032 \\
\hline NLR & $4.0 \pm 3.9$ & $\begin{array}{l}1.057(0.975, \\
1.146)\end{array}$ & 0.178 \\
\hline
\end{tabular}

Note. SD: Standard deviation; HR: Hazard ratio; Cl: Confidence interval; TACE: Transcatheter arterial chemoembolization; ECOG: Eastern Cooperative Oncology Group; BCLC: Barcelona Clinic Liver Cancer; UP: Untreatable progression; TB: Total bilirubin; PT: Prothrombin time; AST: Aspartate aminotransferase; ALT: Alanine aminotransferase; PLR: Platelet-to-lymphocyte ratio; NLR: Neutrophil-to-lymphocyte ratio.

* Interval time refers to the time interval between TACE and camrelizumab initiation.

Table 2 Multivariate analysis of prognostic factors for progression-free survival

\begin{tabular}{|c|c|c|}
\hline Variables & $\mathrm{HR}(95 \% \mathrm{Cl})$ & $P$ value \\
\hline \multicolumn{3}{|c|}{ Combination therapy } \\
\hline Yes & 1 & \\
\hline No & $3.636(1.565,8.447)$ & 0.003 \\
\hline PLR & $1.001(0.998,1.004)$ & 0.690 \\
\hline
\end{tabular}

Note. HR: Hazard ratio; Cl: Confidence interval; PLR: Platelet-to-lymphocyte ratio; NLR: Neutrophil-tolymphocyte ratio.

TABLE 3 Adverse Events 


\begin{tabular}{|c|c|c|c|c|}
\hline \multirow[t]{2}{*}{ Adverse Event } & \multirow[t]{2}{*}{ All Events } & \multicolumn{3}{|c|}{ CTCAE Grade } \\
\hline & & 1 & 2 & $\geq 3$ \\
\hline RCCEP & $14(34.1 \%)$ & $10(24.4 \%)$ & $4(9.8 \%)$ & $0(0 \%)$ \\
\hline Hypothyroidism & $6(14.6 \%)$ & $6(14.6 \%)$ & $0(0 \%)$ & $0(0 \%)$ \\
\hline Asthenia & $2(4.9 \%)$ & $1(2.4 \%)$ & $1(2.4 \%)$ & $0(0 \%)$ \\
\hline Rash & $1(2.4 \%)$ & $1(2.4 \%)$ & $0(0 \%)$ & $0(0 \%)$ \\
\hline Myositis & $1(2.4 \%)$ & $0(0 \%)$ & $1(2.4 \%)$ & $0(0 \%)$ \\
\hline Pneumonitis & $1(2.4 \%)$ & $0(0 \%)$ & $1(2.4 \%)$ & $0(0 \%)$ \\
\hline
\end{tabular}

Note. CTCAE: Common Terminology Criteria for Adverse Events; RCCEP: Reactive cutaneous capillary endothelial proliferation.

Figures 
89 HCC patients received additional camrelizumab due to UP after initial TACE treatment from May 2019 to January 2021

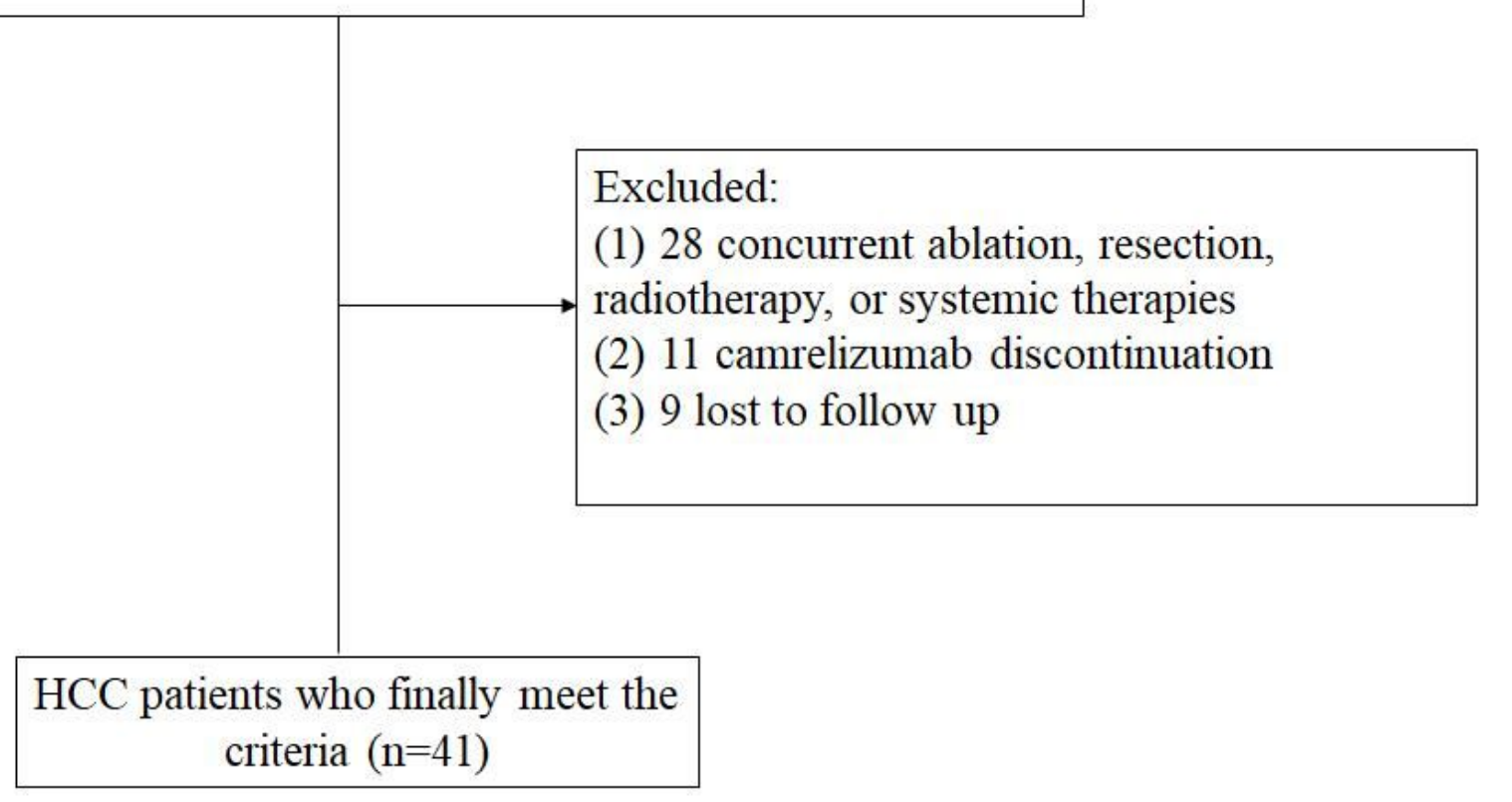

\section{Figure 1}

Flow chart of patients who were included in this study. 


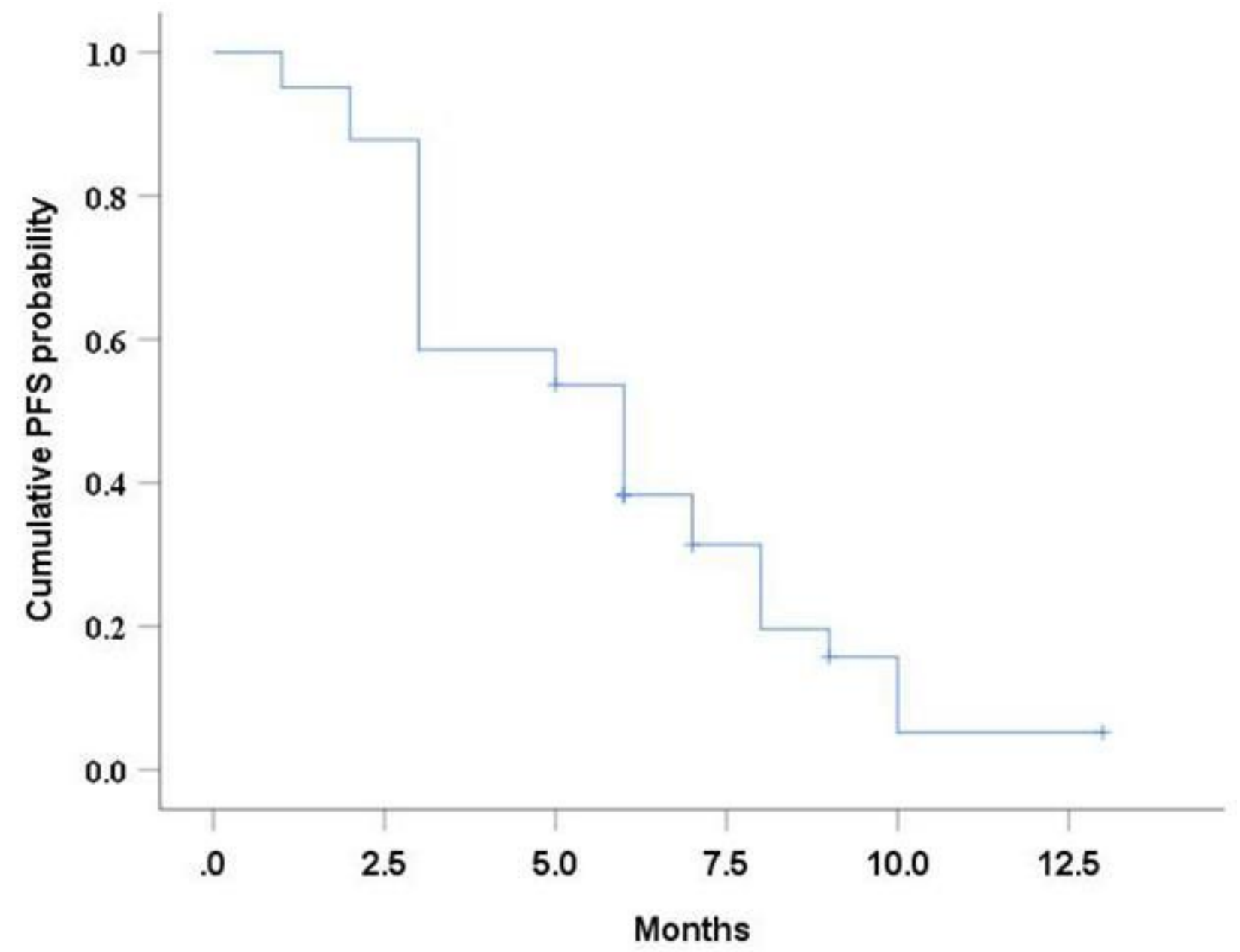

$\begin{array}{lllllll}\text { Number at risk } & 41 & 36 & 21 & 8 & 1 & 1\end{array}$

Figure 2

Kaplan-Meier curve of progression-free survival in HCC patients with untreatable progression after TACE. 


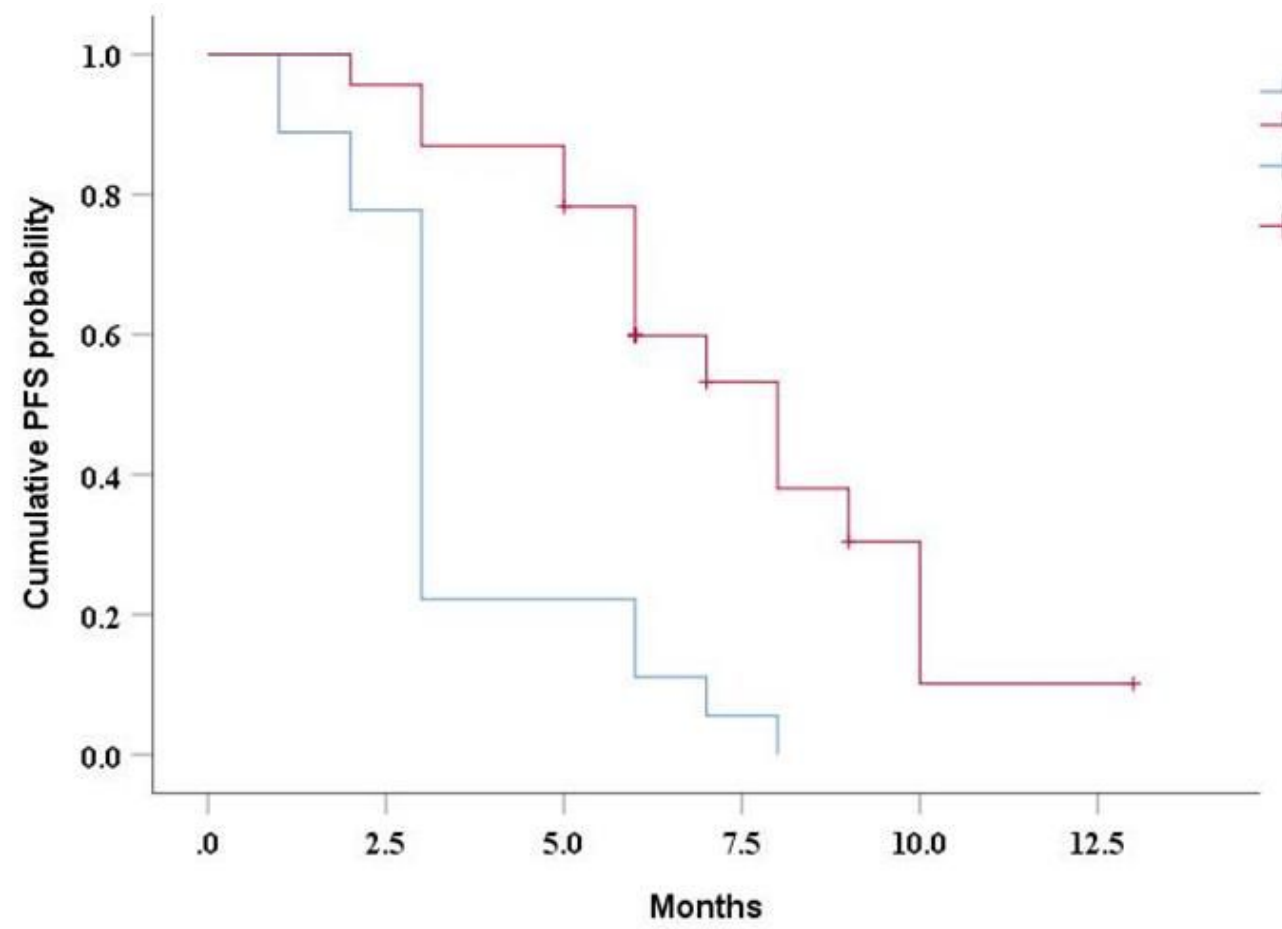

Number at risk

Camrelizumab-TACE $23 \quad 22$

Camrelizumab

$18 \quad 14$

17

4

$7 \quad 1$

1

0

$1 \quad 0 \quad 0$

Figure 3

Kaplan-Meier curves of progression-free survival of two groups of HCC patients with untreatable progression after TACE grouped by treatment method. 